\title{
Coefficients in Series Expansions for Certain Classes of Functions
}

\author{
By P. D. Tuan and David Elliott
}

\begin{abstract}
The problem of evaluating or estimating the coefficients for the expansion of a function in a series of classical orthogonal polynomials is examined. By restricting the functions under consideration to the classes of integral transforms and inverse integral transforms, the coefficients may be expressed in alternative forms which often are more amenable to analysis.
\end{abstract}

1. Introduction. Let $\left\{\phi_{n}(t)\right\}$ be a sequence of functions orthogonal over $[a, b]$ with respect to a positive real-valued function $w(t)$. Suppose that $f(t)$ is a function satisfying certain conditions in order that it may be represented by a uniformly convergent series of the form

$$
f(t)=\sum_{n=0}^{\infty} a_{n} \phi_{n}(t) \quad(a<t<b)
$$

with

$$
a_{n}=\frac{1}{h_{n}} \int_{a}^{b} w(t) \phi_{n}(t) f(t) d t
$$

where $h_{n}$ is the orthogonality constant.

The series expansions of special interest are those in terms of classical orthogonal polynomials. The conditions under which a function may be expanded into a series of these polynomials have been thoroughly investigated, see, for example [1]. The coefficients, on the other hand, are not in general available since their integral representation (1.2) cannot be evaluated in most cases. The knowledge of the coefficients is valuable from a theoretical as well as a practical standpoint. Indeed, we may associate with a function $f(t)$ a formal series without any consideration as to its convergence and its validity as a representation of $f(t)$. Once the coefficients are known explicitly, the resulting series may be studied on its own merits. On the other hand, known values, either exact or approximate, of the coefficients are useful in many problems in numerical analysis, of which the most frequently encountered is the numerical approximation of special functions. The problem of determining the coefficients has received some interest from many authors, see [2] [9]. In these references, the coefficients in the expansions of functions in series of either Jacobi polynomials or their special cases were considered. In particular, Elliott and Szekeres [6] determined the Chebyshev coefficients in the expansion of inverse Laplace transforms; the Laplace and Fourier transforms were considered by Wimp [2]. In [2] and [6], the authors derived alternative

Received November 30, 1970, revised May 21, 1971.

AMS 1970 subject classifications. Primary 42A56, 44A10, 44A15.

Key words and phrases. Coefficients in series expansions of functions, Jacobi polynomials, Fourier transform, generalised function, Laplace transform, Stieltjes transform.

Copyright $\odot 1972$, American Mathematical Society 
expressions for the coefficients in terms of the transforms. These results are in fact special cases of two general formulations, one caters to the inverse integral transforms while the other covers the integral transforms. It is the purpose of this paper to present techniques by which the coefficients in the expansions of integral transforms and inverse integral transforms may be determined, the expansions being in series of any classical orthogonal polynomials. Our techniques which lean more heavily on the results of classical theories and, in one particular instance, on the theory of generalised functions, offer a more general approach to the coefficient problem.

Some preliminary remarks and notations are given in Section 2. Sections 3-5 and Sections 6-8 give what we shall term as the inverse integral transform techniques and the integral transform techniques, respectively. We describe the two types of techniques in general terms in Sections 3 and 6 before examining in detail their particular cases in the remaining sections.

2. Preliminaries and Notations. Let $f(t)$ be a function defined over the real line and $K(x, t)$ be a function of two variables $x$ and $t$, then the function $F(x)$ defined by

$$
F(x)=\int_{c}^{d} f(t) K(x, t) d t
$$

is said to be the integral transform of $f(t)$ if the integral in (2.1) converges for some set of values of $x . K(x, t)$ is called the kernel of the integral transformation. Several forms of the kernel may be taken which result in different transformations, see [10], [11].

On the other hand, it is possible, under certain conditions, to determine a solution of the integral equation (2.1) in the form

$$
f(t)=\int_{\gamma}^{\delta} F(x) H(x, t) d x,
$$

where $\gamma$ and $\delta$ are not necessarily restricted to the real line, and $H(x, t)$ is a function of $x$ and $t$ which may or may not be equal to $K(x, t)$. When such a solution exists, $f(t)$ is said to be the inverse integral transform of $F(x)$.

Another concept which we shall need in the subsequent analysis is the convolution of functions. The convolution of two functions $f(t)$ and $g(t)$ over $(c, d)$, generally denoted by $f(t) * g(t)$, is defined to be the function $h(y)$ given by

$$
h(y)=\int_{0}^{d} f(t) g(y-t) d t,
$$

provided the integral converges for certain values of $y$.

Within the framework of integral transformations, one may express, under certain circumstances, the integral in (2.3) in an alternative form in terms of the integral transforms of $f(t)$ and $g(t)$. For example, if $f(t)$ and $g(t)$ are the inverse Fourier transforms of $F(x)$ and $G(x)$, respectively, then we have [12]

$$
\int_{-\infty}^{\infty} f(t) g(y-t) d t=\frac{1}{2 \pi} \int_{-\infty}^{\infty} F(x) G(x) e^{-i y x} d x .
$$

Such equality is called a convolution theorem. It will be seen in Sections 3-5 that the convolution theorems play an important role in our analysis. 
Throughout this paper, we shall denote the integral transform of a function $f(t)$ by $F(x)$ or more specifically by $g[f(t)]$, where $g$ is replaced by the appropriate initial for a particular integral transformation; for example, $\mathfrak{F}[f(t)]$ will denote the Fourier transform of $f(t)$. It is then understood that $f(t)$ is the inverse integral transform of $F(x)$ if it exists.

Let us next define two classes of functions to which our techniques apply.

Definition 1. A function $f(t)$ defined over an interval $(a, b)$ is said to be of the class $A[\mathfrak{g} ;(a, b)]$ if a function $\hat{f}(t)$ may be found such that

(i) $\hat{f}(t)$ is defined over $(c, d) \supseteq(a, b)$ and $\hat{f}(t)=f(t)$ for $a<t<b$,

(ii) the integral transform $\hat{F}(x)$ of $\hat{f}(t)$ exists.

We note that if $f(t)$ is a function defined over $(c, d)$ and if the integral transform of $f(t)$ exists, then $f(t)$ belongs to $A[g ;(a, b)]$; in this case we have $f(t)=f(t)$ for $c<t<d$.

Definition 2. A function $F(t)$ is said to be of the class $B[g ;(a, b)]$ if $F(t)$ is the integral transform of a function $f(u)$, at least, for all values of $t$ for which $a<t<b$.

Finally, we assume throughout that the function under consideration has an expansion given by (1.1) and (1.2).

3. The Inverse Integral Transform Techniques. Let $f(t)$ be a function of the class $A[g ;(a, b)]$. The coefficients $a_{n}$ for $f(t)$ may be written in the form

$$
a_{n}=\frac{1}{h_{n}} \int_{c}^{d} \hat{f}(t) s_{n}(t) d t
$$

for $n=0,1,2, \cdots$, if we define

$$
\begin{aligned}
s_{n}(t) & =w(t) \phi_{n}(t) & & (a<t<b), \\
& =0 & & (t<a, t>b) .
\end{aligned}
$$

From the definition of $s_{n}(t)$, it is obviously immaterial how $\hat{f}(t)$ is defined outside the interval $(a, b)$. All we require is that its integral transform $\hat{F}(x)$ should exist.

Suppose that the integral transform $\mathscr{g}\left[s_{n}(t)\right]$ exists. Suppose further that there is a convolution theorem of the type (2.4) for the particular integral transformation concerned. Then, it may be possible to write (3.1) in the form of an integral involving the integral transforms of $s_{n}(t)$ and $\hat{f}(t)$. A general expression for this integral cannot be explicitly given here since not only is it dependent upon the particular integral transformation but also upon each orthogonal system $\left\{\phi_{n}(t)\right\}$.

In the next two sections, we shall examine in some detail two special cases in which the transformations concerned are, respectively, of Fourier and Laplace types. In each case, the problem we shall consider is that of determining the coefficients $a_{n}$ in the expansion in series of shifted Jacobi polynomials,

$$
f(t)=\sum_{n=0}^{\infty} a_{n} \Theta_{n}^{(\alpha, \beta)}(t) \quad(\alpha>-1, \beta>-1),
$$

where

$$
{P_{n}^{(\alpha, \beta)}}^{(t)}=P_{n}^{(\alpha, \beta)}\left(\frac{2}{b-a} t-\frac{b+a}{b-a}\right) \quad(a \leqq t \leqq b) .
$$


The weight function for the orthogonal system $\left\{\rho_{n}^{(\alpha, \beta)}(t)\right\}$ is

$$
w(t)=(b-t)^{\alpha}(t-a)^{\beta},
$$

and the coefficients $a_{n}$ are defined by

$$
\begin{aligned}
a_{n}= & \frac{(2 n+\alpha+\beta+1) \Gamma(n+1) \Gamma(n+\alpha+\beta+1)}{(b-a)^{\alpha+\beta+1} \Gamma(n+\alpha+1) \Gamma(n+\beta+1)} \\
& \times \int_{a}^{b}(b-t)^{\alpha}(t-a)^{\beta} \mathscr{P}_{n}^{(\alpha, \beta)}(t) f(t) d t .
\end{aligned}
$$

Similar results may be derived for other systems of classical orthogonal polynomials, for details we refer the reader to [13].

4. The Inverse Fourier Transform Technique. We derive in Theorem 1 an alternative expression for the coefficients $a_{n}$. Theorem 2 gives an extension of the technique through the use of generalised functions. The results will be illustrated by means of some particular examples of interest.

THEOREM 1. Let $f(t)$ be a function of the class $A[F ;(a, b)]$. Then the Jacobi coefficients for $f(t)$ are defined by

$$
\begin{aligned}
a_{n}= & \frac{2^{n} i^{n} \Gamma(n+\alpha+\beta+1)}{\pi(b-a) \Gamma(2 n+\alpha+\beta+1)} \\
& \quad \times \int_{-\infty}^{\infty} \hat{F}\left(\frac{2 x}{b-a}\right) x^{n} e^{2 i a x /(b-a)}{ }_{1} F_{1}(n+\alpha+1 ; 2 n+\alpha+\beta+2 ; 2 i x) d x,
\end{aligned}
$$

for $n=0,1,2, \cdots$, where ${ }_{1} F_{1}(k ; l ; x)$ denotes a confluent hypergeometric function (see [14]).

Proof. Define the function $s_{n}(t)$ by

$$
\begin{aligned}
s_{n}(t) & =(b-t)^{\alpha}(t-a)^{\beta} P_{n}^{(\alpha, \beta)}(t) & & (a<t<b), \\
& =0 & & (t<a, t>b) .
\end{aligned}
$$

The Jacobi coefficients for $f(t)$ may be written as

$$
a_{n}=\frac{(2 n+\alpha+\beta+1) \Gamma(n+1) \Gamma(n+\alpha+\beta+1)}{(b-a)^{\alpha+\beta+1} \Gamma(n+\alpha+1) \Gamma(n+\beta+1)} \int_{-\infty}^{\infty} s_{n}(t) \hat{f}(t) d t .
$$

Let $S_{n}(x)$ denote the Fourier transform of $S_{n}(t)$. By a special case of the convolution theorem for Fourier transforms, namely Parseval's theorem (see [12]), we have

$$
\int_{-\infty}^{\infty} s_{n}(t) \hat{f}(t) d t=\frac{1}{2 \pi} \int_{-\infty}^{\infty} S_{n}(-x) \hat{F}(x) d x .
$$

From [10, p. 123, Eq. (4)], we deduce that

$$
\begin{aligned}
S_{n}(x)= & (b-a)^{n+\alpha+\beta+1}(-1)^{n} i^{n} \frac{\Gamma(n+\alpha+1) \Gamma(n+\beta+1)}{\Gamma(n+1) \Gamma(2 n+\alpha+\beta+2)} \\
& \times x^{n} e^{-i a x}{ }_{1} F_{1}(n+\alpha+1 ; 2 n+\alpha+\beta+2 ;-i(b-a) x) .
\end{aligned}
$$

From (4.3), (4.4) and (4.5), the result as given by (4.1) follows after a simple change of variable. 
It can be seen that the inverse Fourier transform technique depends upon finding a function $\mathfrak{f}(t)$ which, as well as being equal to $f(t)$ for $a<t<b$, also possesses a Fourier transform. Thus, if we consider $f(t)=t^{N}, a<t<b$, where $N$ is a nonnegative integer, and choose $\hat{f}(t)=t^{N}$ for $-\infty<t<\infty$, then, the Fourier transform of $f(t)$ does not exist. However, from the theory of generalised functions [15], it is well known that every generalised function possesses a Fourier transform. In particular, $t^{N}$, when treated as a generalised function, possesses a Fourier transform, and is equal to the ordinary function over the finite interval $(a, b)$. Thus, we would like to extend Theorem 1 so that generalised functions are also included. In the following theorem and its applications, we shall employ the definitions and associated theorems as given in Jones [15].

THEOREM 2. Let $f(t)$ be a function of the class $A[\Im ;(a, b)]$, where $f(t)$ is a generalised function in $K_{a}$ which is equal to the ordinary function $f(t)$ in $(a, b)$, and its Fourier transform $\hat{F}(x)$ is a generalised function in $L_{p}^{\prime}, 1 / p+1 / q=1$ and $1<p \leqq 2$. Then, the Jacobi coefficients for $f(t)$ are defined by

$$
a_{n}=\frac{(2 n+\alpha+\beta+1) \Gamma(n+1) \Gamma(n+\alpha+\beta+1)}{2 \pi(b-a)^{\alpha+\beta+1} \Gamma(n+\alpha+1) \Gamma(n+\beta+1)}\left[\hat{F}(x) * S_{n}(x)\right]_{y=0},
$$

where for each $n=0,1,2, \cdots, \hat{F}(x) * S_{n}(x)$ is a function of $y$ and $S_{n}(x)$ is as defined by (4.5).

Proof. Since $\alpha>-1, \beta>-1$, it is clear that the function $s_{n}(t)$, as defined by (4.2), is in $K_{p}, 1 \leqq p \leqq 1+\epsilon$ for some $\epsilon>0$. If we further restrict $\alpha$ and $\beta$, say $\alpha \geqq-\frac{1}{2}, \beta \geqq-\frac{1}{2}$, then $s_{n}(t) \in K_{p}, 1 \leqq p<2$. On the other hand, from [14, p. 59, Eq. (4.1.3)], we have, for large $|x|$,

$$
{ }_{1} F_{1}(k ; l ;-x)=x^{-k} \frac{\Gamma(l)}{\Gamma(l-k)}\left\{1+O\left(|x|^{-1}\right)\right\} .
$$

It follows that $S_{n}(x)=O\left(x^{-\alpha-1}\right)$ as $|x| \rightarrow \infty$. Since $\alpha>-1$, we have $S_{n}(x) \in L_{a}$, $q>1$, and therefore, $S_{n}(x) \in L_{a}^{\prime}, q>1$. Hence, if $\hat{F}(x) \in L_{p}^{\prime}$ and $\hat{f}(t) \in K_{a}$, by putting $g_{1}=\hat{F}(x)$ and $g_{2}=S_{n}(x)$ and using the fact that $2 \pi \hat{f}(-t)=\mathscr{F}[\hat{F}(x)]$ and $2 \pi S_{n}(-t)=\mathscr{F}\left[S_{n}(x)\right]$, we have from [15, Theorem 6.14] that

$$
\mathfrak{F}\left[\hat{F}(x) * S_{n}(x)\right]=4 \pi^{2} \hat{f}(-t) s_{n}(-t),
$$

or alternatively,

$$
\hat{F}(x) * S_{n}(x)=2 \pi \mathcal{F}\left[\hat{f}(t) s_{n}(t)\right] .
$$

It follows from the definition of $\hat{f}(t)$ and $s_{n}(t)$, and (4.7) that

$$
\hat{F}(x) * S_{n}(x)=2 \pi \int_{a}^{b} f(t)(b-t)^{\alpha}(t-a)^{\beta} P_{n}^{(\alpha, \beta)}(t) e^{-i t y} d t
$$

The left-hand side of (4.8) gives rise to a generalised function which agrees with the ordinary function defined by the right-hand side. Upon identifying these two entities and setting $y=0$, we obtain the required result.

It is important to note that the convolution $\hat{F}(x) * S_{n}(x)$ should be treated in the generalised sense unless $\hat{F}(x)$ is equal to an ordinary function, in which case the 
convolution may be interpreted as the integral $\int_{-\infty}^{\infty} \hat{F}(x) S_{n}(y-x) d x$, and (4.6) reverts to (4.1).

The expressions for the Jacobi coefficients in Theorems 1 and 2 are reduced to fairly simple forms when $\alpha$ and $\beta$ assume special values. In particular, when $\alpha=\beta$, the confluent hypergeometric function ${ }_{1} F_{1}(n+\alpha+1 ; 2 n+2 \alpha+2 ; 2 i x)$ may be expressed in terms of a Bessel function (see [16, Eq. (9.1.69)]). Theorem 1 then essentially covers the results obtained by Wimp [2] but Theorem 2 enables us to apply the technique to a considerably larger range of functions.

The factors $x^{n}$ and $e^{2 i a x /(b-a)}$ in (4.1) indicate that the integral may be reduced to a known Mellin or Fourier transform. For the particular case $\alpha=\beta$, the appearance of the Bessel function in the integrand means that $a_{n}$ may also be evaluated as a Hankel transform.

We shall now consider some examples of the use of Theorem 2. For simplicity, we shall consider the coefficients in the expansion of a function in series of Chebyshev polynomials $T_{n}(t),-1 \leqq t \leqq 1$. Putting $\alpha=\beta=-\frac{1}{2}$ in (4.1) and (4.6) and adjusting the orthogonality constant, we obtain the Chebyshev coefficients as

$$
a_{n}=\frac{i^{n}}{\pi} \int_{0}^{\infty}\left[\hat{F}(x)+(-1)^{n} \hat{F}(-x)\right] J_{n}(x) d x,
$$

and when $f(t)$ is a generalised function,

$$
a_{n}=\frac{(-1)^{n} i^{n}}{\pi}\left[\hat{F}(x) * J_{n}(x)\right]_{y=0} .
$$

We again note that (4.10) reverts to (4.9) when $\hat{F}(x)$ equals an ordinary function.

Let us first consider the function $f(t)=(1-t)^{\gamma}$, where $\gamma$ is a positive real number not an integer. We shall choose $f^{f}(t)$ to be the generalised function $(1-t)^{\gamma} H(1-t)$ where $H(t)$ denotes the Heaviside step function. From [15, p. 469 and Theorem 3.10], we have

$$
\hat{F}(x)=\Gamma(\gamma+1) \exp \left[\frac{1}{2} i \pi(\gamma+1) \operatorname{sgn} x-i x\right]|x|^{-\gamma-1} .
$$

Since $(1-t)^{\gamma} H(1-t) \in K_{a}, q>1$, for $\gamma>0$, and $\hat{F}(x) \in L_{p}^{\prime}, 1 / p+1 / q=1$, Theorem 2 applies. Furthermore, $\hat{F}(x)$ is equal to the ordinary function denoted by the same symbol, and we have

$$
\begin{aligned}
a_{n}=\frac{2(-1)^{n} \Gamma(\gamma+1)}{\pi}\left\{\cos \phi \int_{0}^{\infty} x^{-\gamma-1} \cos x J_{n}(x) d x\right. & \\
& \left.-\sin \phi \int_{0}^{\infty} x^{-\gamma-1} \sin x J_{n}(x) d x\right\},
\end{aligned}
$$

where $\phi=\frac{1}{2}(n-\gamma-1) \pi$. These two integrals may be evaluated in closed form $[11$, p. $33, \mathrm{Eq} .(7)]$, to give after some algebra

$$
a_{n}=-\frac{2^{1-\gamma} \sin (\pi \gamma) \Gamma(n-\gamma) \Gamma(2 \gamma+1)}{\pi \Gamma(n+\gamma+1)},
$$

provided $0<\gamma<n$. For large $n, a_{n}=O\left(n^{-2 \gamma-1}\right)$.

The Chebyshev coefficients for the function $(1+t)^{\gamma}$ may be similarly deduced to 
be

$$
a_{n}=\frac{(-1)^{n+1} 2^{1-\gamma} \sin (\pi \gamma) \Gamma(n-\gamma) \Gamma(2 \gamma+1)}{\pi \Gamma(n+\gamma+1)} \quad(0<\gamma<n),
$$

which, for large $n$, is $O\left(n^{-2 \gamma-1}\right)$.

We can proceed in an analogous fashion for functions with an algebraic singularity at any other point inside or outside $[-1,1]$. We shall discuss below a technique for dealing with functions possessing two or more singularities.

We first note that if $f(t)$ is of the form $(1-t)^{\gamma} g(t)$ or $(1+t)^{\gamma} g(t)$, where $g(t)$ is a function possessing a Taylor series with nonzero radius of convergence about $t=1$ and $t=-1$ in the respective cases, then it may be shown that their Chebyshev coefficients are given, respectively, by

$$
a_{n}=-\frac{g(1) 2^{1-\gamma} \sin (\pi \gamma) \Gamma(2 \gamma+1)}{\pi n^{2 \gamma+1}}\left\{1+O\left(n^{-2}\right)\right\},
$$

and

$$
a_{n}=\frac{(-1)^{n+1} g(-1) 2^{1-\gamma} \sin (\pi \gamma) \Gamma(2 \gamma+1)}{\pi n^{2 \gamma+1}}\left\{1+O\left(n^{-2}\right)\right\},
$$

for large $n$ and $0<\gamma<n$.

Suppose now that we have a function, say

$$
f(t)=(1-t)^{\gamma}(1+t)^{\delta} g(t) \quad(-1 \leqq t \leqq 1),
$$

where $\gamma$ and $\delta$ are positive real numbers not integers. The results (4.13) and (4.14) would immediately apply if we could separate the singularities at $t= \pm 1$. In other words, if we could find functions $g_{1}(t)$ and $g_{2}(t)$ having a Taylor series with nonzero radius of convergence about 1 and -1 , respectively, such that

$$
f(t)=(1-t)^{\gamma} g_{1}(t)+(1+t)^{\delta} g_{2}(t) \quad(-1 \leqq t \leqq 1),
$$

then an asymptotic estimate for the coefficients may be obtained by superposing the contributions from the singularities.

We present here a technique by which the separation of singularities may be achieved. Let us consider a function of the form

$$
f(t)=g(t) \prod_{i=1}^{m}\left|t-t_{i}\right|^{\gamma /} \quad(-1 \leqq t \leqq 1),
$$

where $t_{1}=-1, t_{i}<t_{i+1}, t_{m}=1$, and $\gamma_{i}, i=1,2, \cdots, m$, are positive real numbers not integers.

Let $t_{0}$ and $t_{m+1}$ be two arbitrary points such that $t_{0}<t_{1}$ and $t_{m+1}>t_{m}$. We define, for $i=1,2, \cdots, m$, the functions

$$
\begin{aligned}
\theta_{i}(t) & =0 & & \left(t \leqq t_{i-1}\right), \\
& =\tau_{i-1}(t) & & \left(t_{i-1}<t \leqq t_{i}\right), \\
& =1-\tau_{i}(t) & & \left(t_{i}<t \leqq t_{i+1}\right), \\
& =0 & & \left(t>t_{i+1}\right),
\end{aligned}
$$

where 


$$
\tau_{i-1}(t)=\frac{\int_{t_{i-1}}^{t} \exp \left[-1 /\left(t-t_{i-1}\right)\left(t_{i}-t\right)\right] d t}{\int_{t_{i-1}}^{t_{i}} \exp \left[-1 /\left(t-t_{i-1}\right)\left(t_{i}-t\right)\right] d t} \quad\left(t_{i-1} \leqq t \leqq t_{i}\right) .
$$

It can be readily verified that

(i) $\theta_{i}(t)$ is infinitely differentiable for all $t$ and $i=1,2, \cdots, m$.

(ii) $\theta_{i}\left(t_{i}\right)=1, \theta_{i}^{(k)}\left(t_{i}\right)=0$, for $i=1,2, \cdots, m$ and all positive integers $k$.

(iii) $\theta_{i}^{(k)}\left(t_{i-1}\right)=\theta_{i}^{(k)}\left(t_{i+1}\right)=0$, for $i=1,2, \cdots, m$ and all nonnegative integers $k$.

(iv) $\sum_{i=1}^{m} \theta_{i}(t)=1$, for $-1 \leqq t \leqq 1$.

Making use of the functions $\theta_{i}(t)$ we may write the function $f(t)$ as

$$
f(t)=\sum_{i=1}^{m}\left|t-t_{i}\right|^{\gamma_{i}} g_{i}(t)
$$

where

$$
g_{i}(t)=g(t) \theta_{i}(t) \prod_{i=1 ; i \neq i}^{m}\left|t-t_{i}\right|^{\gamma_{i}}
$$

Each of the functions $\left|t-t_{i}\right|^{\gamma_{i}} g_{i}(t), i=1,2, \cdots, m$, has exactly one singularity at $t=t_{i}$. Furthermore, each $g_{i}(t), i=1,2, \cdots, m$, can be expanded in a series with nonzero radius of convergence about the point $t=t_{i}$. The expressions (4.18) and (4.19) thus give us the required result.

As an example, let us estimate the Chebyshev coefficients for the function $f(t)=$ $\left(1-t^{2}\right)^{1 / 2}$. We have, in this case,

$$
f(t)=(1-t)^{1 / 2} g_{1}(t)+(1+t)^{1 / 2} g_{2}(t),
$$

with

$$
g_{1}(t)=(1+t)^{1 / 2} \theta_{1}(t) \text { and } g_{2}(t)=(1-t)^{1 / 2} \theta_{2}(t) .
$$

From (4.13), (4.14), and (4.20), we deduce that, for large $n$, the Chebyshev coefficients for $\left(1-t^{2}\right)^{1 / 2}$ are given asymptotically by

$$
a_{n}=-\frac{\sqrt{ } 2}{\pi n^{2}}\left[g_{1}(1)+(-1)^{n} g_{2}(-1)\right]\left[1+O\left(n^{-2}\right)\right] .
$$

Since $g_{1}(1)=g_{2}(-1)=\sqrt{ } 2$, we have finally, for large $m$,

$$
a_{2 m+1}=0 \text { and } a_{2 m}=-\frac{1}{\pi m^{2}}\left[1+O\left(m^{-2}\right)\right] .
$$

These estimates compare favourably with the actual values

$$
a_{2 m+1}=0 \text { and } a_{2 m}=-\frac{1}{\pi\left(m^{2}-\frac{1}{4}\right)} .
$$

5. The Inverse Laplace Transform Technique. In the inverse Laplace transform technique we derive, by means of the convolution theorem for Laplace transforms, an alternative expression for the coefficients in terms of an integral whose range of integration is a line parallel to the imaginary axis in the complex $x$-plane. It is clear from the general technique of Section 3 and Definition 1 that the interval 
$(a, b)$ over which the function is to be expanded must be contained in $(c, d)$, the range of integration of the integral defining the transformation. Consequently, the consideration of an expansion over an arbitrary interval $(a, b)$ would necessitate the use of two types of Laplace transformations, namely the one-sided and the two-sided Laplace transformations. These two integral transformations are defined, respectively, by [17],

$$
\begin{array}{ll}
\mathfrak{L}_{1}[f(t)]=F_{1}(x)=\int_{0}^{\infty} f(t) e^{-x t} d t \quad\left(\operatorname{Re} x>\sigma^{\prime}\right), \\
\mathfrak{L}_{2}[f(t)]=F_{2}(x)=\int_{-\infty}^{\infty} f(t) e^{-x t} d t \quad\left(\sigma^{\prime}<\operatorname{Re} x<\sigma^{\prime \prime}\right),
\end{array}
$$

where $x=\sigma+i \omega, \sigma$ and $\omega$ being real numbers.

The two-sided Laplace transform may be alternatively defined by

$$
F_{2}(x)=F_{1}^{(1)}(x)+F_{1}^{(2)}(-x),
$$

where

$$
\begin{array}{ll}
F_{1}^{(1)}(x)=\int_{0}^{\infty} f(t) e^{-x t} d t=\mathscr{L}_{1}[f(t)] & \left(\operatorname{Re} x>\sigma^{\prime}\right), \\
F_{1}^{(2)}(x)=\int_{0}^{\infty} f(-t) e^{-x t} d t=\mathscr{L}_{1}[f(-t)] & \left(\operatorname{Re} x>-\sigma^{\prime \prime}\right),
\end{array}
$$

provided that $\sigma^{\prime}<\sigma^{\prime \prime}$. In other words, the two-sided Laplace transform may be looked upon as the sum of two one-sided ones whose regions of convergence overlap. This common region is then the region of convergence of the two-sided Laplace transform.

On the other hand, every one-sided Laplace transform is related to a two-sided one through

$$
\mathfrak{L}_{1}[f(t)]=\mathfrak{L}_{2}[f(t) H(t)],
$$

where $H(t)$ is the Heaviside step function.

Finally, a two-sided Laplace transform of a function $f(t)$ converges absolutely on a line $\operatorname{Re} x=\sigma$ if

$$
\int_{-\infty}^{\infty}|f(t)| e^{-\sigma t} d t<\infty
$$

Before going into the actual technique, let us first look at a simple example which may serve as a motivation for the subsequent analysis. Consider the function $f(t)=$ $e^{\gamma t}, \gamma$ real, over the interval $(a, b)$. Since $e^{\gamma t}$ is a function of bounded variation in any finite interval $(a, b)$, say $a<0<b$, a convergent Jacobi series exists such that

$$
e^{\gamma t}=\sum_{n=0}^{\infty} a_{n} P_{n}^{(\alpha, \beta)}(t) \quad(a<t<b)
$$

where

$$
a_{n}=\frac{(2 n+\alpha+\beta+1) \Gamma(n+1) \Gamma(n+\alpha+\beta+1)}{(b-a)^{\alpha+\beta+1} \Gamma(n+\alpha+1) \Gamma(n+\beta+1)} \int_{-\infty}^{\infty} e^{\gamma t} s_{n}(t) d t,
$$

$s_{n}(t)$ being as defined by (4.2). 
It is further known that

$$
\begin{aligned}
F_{1}^{(1)}(x)=\int_{0}^{\infty} e^{\gamma t} e^{-x t} d t=\frac{1}{x-\gamma} \quad(\operatorname{Re} x>\gamma), \\
F_{1}^{(2)}(-x)=\int_{-\infty}^{0} e^{\gamma t} e^{-x t} d t=-\frac{1}{x-\gamma} \quad(\operatorname{Re} x<\gamma) .
\end{aligned}
$$

Since $F_{1}^{(1)}(x)$ and $F_{1}^{(2)}(-x)$ have no common region of convergence, it follows that $\mathscr{L}_{2}\left[e^{\gamma t}\right]$ does not exist. Hence, the general technique of Section 3 does not apply here.

This example suggests that something other than the standard approach is required. We shall deal with functions of this type in Theorem 3. A function possessing a two-sided Laplace transform is treated as a special case of Theorem 3 and the result is summed up in the form of a corollary.

To prove the main result, we need the following lemma.

LEMMA 1. Let the functions $f(t)$ and $g(t)$ be piecewise continuous over $(-\infty, \infty)$. Let the two-sided Laplace transforms $F_{2}(x)=\mathscr{L}_{2}[f(t)]$ and $G_{2}(x)=\mathscr{L}_{2}[g(t)]$ converge absolutely on the same line $\operatorname{Re} x=\sigma$ in the complex $x$-plane. Then the function $h(\tau)$ defined by

$$
h(\tau)=\int_{-\infty}^{\infty} f(t) g(t-\tau) d t
$$

is a continuous function of $\tau$, and furthermore,

$$
h(\tau)=\frac{1}{2 \pi i} \lim _{\omega \rightarrow \infty} \int_{\sigma-i \omega}^{\sigma+i \omega} F_{2}(x) G_{2}(-x) e^{x \tau} d x .
$$

Proof. This lemma is a modified version of Theorem 11.2 in Le Page [17] to which the reader is referred for details.

We are now in the position to state and prove the fundamental result of the inverse Laplace transform technique.

THEOREM 3. Let $f(t)$ be a piecewise continuous function over $(-\infty, \infty)$ such that

(i) $f^{(1)}(t)=f(t) H(t) \in A\left[\mathfrak{L}_{2} ;(-\infty, \infty)\right]$, where $F_{2}^{(1)}(x)=\mathfrak{L}_{2}\left[f^{(1)}(t)\right]$ converges absolutely on some line $\operatorname{Re} x=\sigma_{1}\left(\left|\sigma_{1}\right|<\infty\right)$.

(ii) $f^{(2)}(t)=f(t) H(-t) \in A\left[\mathscr{L}_{2} ;(-\infty, \infty)\right]$, where $F_{2}^{(2)}(x)=\mathscr{L}_{2}\left[f^{(2)}(t)\right]$ converges absolutely on some line $\operatorname{Re} x=\sigma_{2}\left(\left|\sigma_{2}\right|<\infty\right)$.

Then, the Jacobi coefficients for $f(t)$ are defined by

$$
\begin{aligned}
a_{n}= & \frac{(b-a)^{n} \Gamma(n+\alpha+\beta+1)}{2 \pi i \Gamma(2 n+\alpha+\beta+1)} \\
& \times \sum_{i=1}^{2} \lim _{\omega \rightarrow \infty} \int_{\sigma_{j}-i \omega}^{\sigma_{i}+i \omega} F_{2}^{(i)}(x) x^{n} e^{b x} \\
& \quad{ }_{1} F_{1}(n+\alpha+1 ; 2 n+\alpha+\beta+2 ;-(b-a) x) d x,
\end{aligned}
$$

for $n=0,1,2, \cdots$.

Proof. Let us first assume that $a<0<b$. Since

$$
f(t)=f^{(1)}(t)+f^{(2)}(t)=f(t) H(t)+f(t) H(-t) \quad(-\infty<t<\infty) .
$$


the Jacobi coefficients $a_{n}$ for $f(t)$ may be written as

$$
a_{n}=a_{n}^{(1)}+a_{n}^{(2)},
$$

where

$$
a_{n}^{(i)}=\frac{(2 n+\alpha+\beta+1) \Gamma(n+1) \Gamma(n+\alpha+\beta+1)}{(b-a)^{\alpha+\beta+1} \Gamma(n+\alpha+1) \Gamma(n+\beta+1)} \int_{-\infty}^{\infty} f^{(i)}(t) s_{n}(t) d t,
$$

for $j=1,2$, and $s_{n}(t)$ is as defined in (4.2). The existence of $a_{n}$ implies the existence of $a_{n}^{(1)}$ and $a_{n}^{(2)}$ and vice-versa.

From conditions (i) and (ii), $F_{2}^{(1)}(x)$ and $F_{2}^{(2)}(x)$ converge absolutely on the lines $\operatorname{Re} x=\sigma_{1}\left(\left|\sigma_{1}\right|<\infty\right)$ and $\operatorname{Re} x=\sigma_{2}\left(\left|\sigma_{2}\right|<\infty\right)$ respectively. On the other hand, the two-sided Laplace transform $S_{n}(x)$ of $s_{n}(t)$ is given by [18, p. 213, Eq. (4)]

$$
\begin{aligned}
S_{n}(x)= & (-1)^{n}(b-a)^{n+\alpha+\beta+1} \frac{\Gamma(n+\alpha+1) \Gamma(n+\beta+1)}{\Gamma(n+1) \Gamma(2 n+\alpha+\beta+2)} \\
& \times x^{n} e^{-b x} F_{1}(n+\alpha+1 ; 2 n+\alpha+\beta+2 ;(b-a) x),
\end{aligned}
$$

for all (real or complex) values of $x$. Furthermore, $S_{n}(x)$ converges absolutely on any line $\operatorname{Re} x=\sigma(|\sigma|<\infty)$. Thus, we can apply Lemma 1 with $\tau=0$ to the integrals (5.5) to get

$$
\begin{aligned}
a_{n}= & \frac{(2 n+\alpha+\beta+1) \Gamma(n+1) \Gamma(n+\alpha+\beta+1)}{2 \pi i(b-a)^{\alpha+\beta+1} \Gamma(n+\alpha+1) \Gamma(n+\beta+1)} \\
& \times \sum_{i=1}^{2} \lim _{\omega \rightarrow \infty} \int_{\sigma_{j-i \omega}}^{\sigma i+i \omega} F_{2}^{(j)}(x) S_{n}(-x) d x .
\end{aligned}
$$

Equations (5.6) and (5.7) give the required Eq. (5.3).

When $a$ and $b$ are of the same sign, it is obvious from (5.5) and the definition of $s_{n}(t)$ that either $a_{n}^{(1)}$ or $a_{n}^{(2)}$ is zero depending on whether $a$ and $b$ are both negative or both positive respectively. The same analysis is still valid, and (5.3) also holds in this case. More precisely, we have

$$
a_{n}=a_{n}^{(j)}=\frac{(b-a)^{n} \Gamma(n+\alpha+\beta+1)}{2 \pi i \Gamma(2 n+\alpha+\beta+1)}
$$

$$
\begin{aligned}
\times \lim _{\omega \rightarrow \infty} \int_{\sigma_{i-i \omega}}^{\sigma_{i+i \omega}} F_{2}^{(i)}(x) x^{n} e^{b x} \\
\quad \cdot{ }_{1} F_{1}(n+\alpha+1 ; 2 n+\alpha+\beta+2 ;-(b-a) x) d x,
\end{aligned}
$$

where $j=1$ and $j=2$ correspond to the cases $b>a \geqq 0$ and $a<b \leqq 0$ respectively.

It is interesting to note that $F_{2}^{(1)}(x)$ and $F_{2}^{(2)}(x)$ are actually one-sided Laplace transforms of $f(t)$ and when $a$ and $b$ are of the same sign, the knowledge of only one of $F_{2}^{(1)}(x)$ and $F_{2}^{(2)}(x)$ is needed.

An immediate consequence of Theorem 3 is

COROLlARY 1. Let $f(t)$ be a piecewise continuous function over $(-\infty, \infty)$ such that $f(t) \in A\left[\mathfrak{L}_{2} ;(-\infty, \infty)\right]$ and $F_{2}(x)=\mathfrak{L}_{2}[f(t)]$ converges absolutely on some line $\operatorname{Re} x=$ $\sigma(|\sigma|<\infty)$. Then the Jacobi coefficients for $f(t)$ are defined by 


$$
\begin{aligned}
a_{n}= & \frac{(b-a)^{n} \Gamma(n+\alpha+\beta+1)}{2 \pi i \Gamma(2 n+\alpha+\beta+1)} \\
& \times \lim _{\omega \rightarrow \infty} \int_{\sigma-i \omega}^{\sigma+i \omega} F_{2}(x) x^{n} e^{b x} \\
& \quad{ }_{1} F_{1}(n+\alpha+1 ; 2 n+\alpha+\beta+2 ;-(b-a) x) d x,
\end{aligned}
$$

for $n=0,1,2, \cdots$.

Proof. If $\mathfrak{L}_{2}[f(t)]$ exists then, by definition, $\mathfrak{L}_{2}[f(t) H(t)]$ and $\mathfrak{L}_{2}[f(t) H(-t)]$ must also exist and their regions of convergence overlap and we can write

$$
\mathfrak{L}_{2}[f(t)]=\mathscr{L}_{2}[f(t) H(t)]+\mathfrak{L}_{2}[f(t) H(-t)],
$$

for $\sigma^{\prime}<\operatorname{Re} x<\sigma^{\prime \prime}$ say. Furthermore, the absolute convergence of $\mathfrak{L}_{2}[f(t)]$ on some line $\operatorname{Re} x=\sigma(|\sigma|<\infty)$ is a necessary and sufficient condition for $\mathscr{L}_{2}[f(t) H(t)]$ and $\mathfrak{L}_{2}[f(t) H(-t)]$ to converge on the same line. Thus, $f(t)$ satisfies the conditions of Theorem 3, and (5.9) follows from (5.3) with $\sigma_{1}=\sigma_{2}=\sigma$, together with (5.10).

Putting $\alpha=\beta=-\frac{1}{2}$ in (5.3) and adjusting the orthogonality constant, yield the results obtained by Elliott and Szekeres [6].

Let us now consider some examples of the application of Theorem 3. We first return to the preliminary example $f(t)=e^{\gamma t}, \gamma$ real. $F_{2}^{(1)}(x)=\mathscr{L}_{2}\left[e^{\gamma t} H(t)\right]$ and $F_{2}^{(2)}(x)$ $=\mathscr{L}_{2}\left[e^{\gamma t} H(-t)\right]$ are given, respectively, by (5.1) and (5.2). Moreover, one could readily verify that $F_{2}^{(1)}(x)$ converges absolutely on any line $\operatorname{Re} x=\sigma_{1}, \gamma<\sigma_{1}<\infty$, and so does $F_{2}^{(2)}(x)$ on any line $\operatorname{Re} x=\sigma_{2},-\infty<\sigma_{2}<\gamma$. Theorem 3 applies to the function $e^{\gamma t}$ to give

$$
a_{n}=\frac{(b-a)^{n} \Gamma(n+\alpha+\beta+1)}{2 \pi i \Gamma(2 n+\alpha+\beta+1)} \lim _{\omega \rightarrow \infty}\left\{\int_{\sigma_{1}-i \omega}^{\sigma_{1}+i \omega}-\int_{\sigma_{2}-i \omega}^{\sigma_{2}+i \omega}\right\} \phi(x) d x,
$$

where

$$
\phi(x)=x^{n} e^{b x} F_{1}(n+\alpha+1 ; 2 n+\alpha+\beta+2 ;-(b-a) x) /(x-\gamma) .
$$

To evaluate the integrals on the right-hand side of (5.11), we consider the contour integral $\int_{e} \phi(x) d x$, where the contour $\mathcal{e}$ is chosen to be the rectangle described in the positive (anticlockwise) direction in the complex $x$-plane, with vertices at the points $\sigma_{i} \pm i \omega, j=1,2$. Letting $\omega \rightarrow \infty$, it could be shown that the contributions from the horizontal sides of the rectangular contour vanish and we have

$$
\int_{e} \phi(x) d x=\lim _{\omega \rightarrow \infty}\left\{\int_{\sigma_{1}-i \omega}^{\sigma_{1}+i \omega}-\int_{\sigma_{2}-i \omega}^{\sigma_{2}+i \omega}\right\} \phi(x) d x .
$$

Since $\phi(x)$ has only a simple pole at $x=\gamma$, we have, from the theory of residues,

$$
\int_{e} \phi(x) d x=\gamma^{n} e^{b \gamma}{ }_{1} F_{1}(n+\alpha+1 ; 2 n+\alpha+\beta+2 ;-(b-a) \gamma) .
$$

From (5.11), (5.13), and (5.14) we obtain, finally,

$$
a_{n}=\frac{(b-a)^{n} \Gamma(n+\alpha+\beta+1)}{\Gamma(2 n+\alpha+\beta+1)} \gamma^{n} e^{b \gamma}
$$

$$
{ }_{1} F_{1}(n+\alpha+1 ; 2 n+\alpha+\beta+2 ;-(b-a) \gamma) .
$$


As a further illustration of the inverse Laplace transform technique, we consider the function $e^{-\Lambda / t}, A>0$. We wish to find the coefficients $a_{n}$ in the expansion for $e^{-A / t}$ in a series of $T_{n}^{*}(t)=T_{n}(2 t-1), 0 \leqq t \leqq 1$. The two-sided Laplace transform of $e^{-\Lambda / t} H(t)$ exists and is given by, [10, p. 146, Eq. (25)],

$$
\mathfrak{L}_{2}\left[e^{-A / t} H(t)\right]=2 A^{1 / 2} x^{-1 / 2} K_{1}\left(2 A^{1 / 2} x^{1 / 2}\right),
$$

where $K_{m}(x)$ is the modified Bessel function of the second kind of order $m$. It can also be easily checked that $\mathcal{L}_{2}\left[e^{-\Lambda / t} H(t)\right]$ converges absolutely on any line $\operatorname{Re} x=\sigma_{1}$, $0<\sigma_{1}<\infty$. Theorem 3 gives in this case

$$
a_{n}=\frac{2 A^{1 / 2}}{\pi i} \lim _{\omega \rightarrow \infty} \int_{\sigma_{1}-i \omega}^{\sigma_{1}+i \omega} x^{-1 / 2} K_{1}\left(2 A^{1 / 2} x^{1 / 2}\right) e^{x / 2} I_{n}\left(\frac{1}{2} x\right) d x .
$$

In order to evaluate this integral, let us consider the contour integral

$$
\int_{\mathfrak{e}} \phi(x) d x=\int_{\mathfrak{e}} x^{-1 / 2} K_{1}\left(2 A^{1 / 2} x^{1 / 2}\right) e^{x / 2} I_{n}\left(\frac{1}{2} x\right) d x,
$$

where $\mathfrak{C}$ denotes a closed contour described in the positive direction in the complex $x$-plane cut along the negative real axis. $\mathcal{C}$ consists of

(i) a line segment $A B$ parallel to the imaginary axis joining the point $A$, $x=\sigma_{1}-i R$, to the point $B, x=\sigma_{1}+i R$, where $R>\sigma_{1}>0$,

(ii) an arc $B C$, with radius $R$ and centre $x=\sigma_{1}$, joining $B$ and the point $C, x=\left(R-\sigma_{1}\right) e^{i \pi}$,

(iii) a line segment $C D$ from $C$ to the point $D, x=r e^{i r}, 0<r<\sigma_{1}$,

(iv) a circle $D E$, with radius $r$ and centre $x=0$, joining $D$ to the point $E, x=r e^{-i \pi}$,

(v) a line segment $E F$ from $E$ to the point $F, x=\left(R-\sigma_{1}\right) e^{-i \pi}$,

(vi) an arc $F A$ with radius $R$ and centre $x=\sigma_{1}$.

Since the integrand $\phi(x)$ is analytic on and within the contour $\mathcal{C}$, we have from Cauchy's theorem that

$$
\int_{e} \phi(x) d x=0
$$

Letting $R \rightarrow \infty$ and $r \rightarrow 0$, it could be shown that the contributions to the contour integral (5.17) from the circular arcs vanish. We thus have

$$
\lim _{\omega \rightarrow \infty} \int_{\sigma_{1}-i \omega}^{\sigma_{1}+i \omega} \phi(x) d x=\lim _{R \rightarrow \infty} \int_{A B} \phi(x) d x=-\lim _{R \rightarrow \infty ; r \rightarrow 0}\left\{\int_{C D}+\int_{B P}\right\} \phi(x) d x .
$$

On $C D$ and $E F$, one may write $x=u e^{i \pi}$ and $x=u e^{-i \pi}$, respectively, where $0<u<\infty$. Combining the two integrals on the right-hand side of (5.19), with $\phi(x)$ as defined in (5.17), we obtain, after some algebra,

$$
a_{n}=4(-1)^{n+1} A^{1 / 2} \int_{0}^{\infty} e^{-v>/ 2} I_{n}\left(\frac{1}{2} v^{2}\right) J_{1}\left(2 A^{1 / 2} v\right) d v
$$

The integral in (5.20) is of Hankel form, and from [11, p. 68, Eq. (8)] we have finally

$$
a_{n}=\frac{2(-1)^{n+1}}{\pi^{1 / 2}} G_{23}^{21}\left(A \mid \begin{array}{l}
1-n, 1+n \\
1, \frac{1}{2}, 0
\end{array}\right),
$$


where

$$
G_{p q}^{m n}\left(x \mid \begin{array}{l}
a_{1}, a_{2}, \cdots, a_{p} \\
b_{1}, b_{2}, \cdots, b_{q}
\end{array}\right)
$$

denotes a Meijer $G$-function (see [19, p. 206]). The coefficients $a_{n}$, as given by (5.21), are exact. Asymptotic estimates of $a_{n}$ for large $n$ have been obtained by Miller [7].

6. The Integral Transform Technique. Let $F(t)$ be a function of the class $B[g ;(a, b)]$. The coefficients for $F(t)$ are thus defined by

$$
a_{n}=\frac{1}{h_{n}} \int_{a}^{b} d t \int_{c}^{d} f(u) K(t, u) w(t) \phi_{n}(t) d u .
$$

Under suitable conditions, we can invert the order of integration in (6.1) to get

$$
a_{n}=\frac{1}{h_{n}} \int_{c}^{d} f(u) S_{n}(u) d u,
$$

where

$$
S_{n}(u)=\int_{a}^{b} w(t) \phi_{n}(t) K(t, u) d t
$$

We note that if $(a, b) \subseteq(c, d)$ and if we define $s_{n}(t)$ as in (3.2), then $S_{n}(u)$ is in fact the integral transform of $s_{n}(t)$.

Eqs. (6.2) and (6.3) give us an alternative representation for the coefficients $a_{n}$.

It is seen from above that the technique is based on the invertibility of the iterated integral in (6.1). The conditions under which a change of the order of integration may be carried out have been set out in [20, Chapter I] to which the reader is referred for further details. More general conditions are given by Fubini's theorem, see, for example, [21]. For convenience, we define the class of functions $C[(a, b) ;(c, d)]$ as follows.

Definition 3. A function $\phi(t, u)$ is said to be of the class $C[(a, b) ;(c, d)]$ if

$$
\int_{a}^{b} d t \int_{c}^{d} \phi(t, u) d u=\int_{0}^{d} d u \int_{a}^{b} \phi(t, u) d t
$$

where $a, b, c$, and $d$ are real numbers finite or infinite.

In the next two sections, we shall restate this technique in two special cases in which the integral transforms are of Laplace type and Stieltjes type respectively. In both cases, we wish to determine the Jacobi coefficients as defined by (3.6). The results for the coefficients in the expansions in series of other classical orthogonal polynomials may be obtained in an analogous manner (see [13]).

We finally note that if $F(t)$ is the Fourier transform of a function $f(u)$ then $2 \pi f(-u)$ is the Fourier transform of $F(t)$. Thus, $F(t)$ may be looked upon as one whose Fourier transform exists; such functions have already been considered in Section 4.

7. The Laplace Transform Technique. Consider a function of the class $B\left[\varrho_{1} ;(a, b)\right]$, i.e., a function which satisfies 


$$
F(t)=\mathscr{L}_{1}[f(u)]=\int_{0}^{\infty} e^{-u t} f(u) d u \quad(a<t<b) .
$$

The Jacobi coefficients for $F(t)$ are given in the following theorem.

THEOREM 4. Let $F(t)$ be a function such that

(i) $F(t) \in B\left[\mathfrak{L}_{1} ;(a, b)\right]$, where $F(t)=\mathfrak{L}_{1}[f(u)]$.

(ii) $f(u) e^{-t u}(b-t)^{\alpha}(t-a)^{\beta} \mathbb{P}_{n}^{(\alpha, \beta)}(t) \in C[(a, b) ;(0, \infty)]$.

Then the Jacobi coefficients for $F(t)$ are defined by

$$
\begin{aligned}
a_{n}= & \frac{(-1)^{n}(b-a)^{n} \Gamma(n+\alpha+\beta+1)}{\Gamma(2 n+\alpha+\beta+1)} \\
& \times \int_{0}^{\infty} f(u) u^{n} e^{-b u}{ }_{1} F_{1}(n+\alpha+1 ; 2 n+\alpha+\beta+2 ;(b-a) u) d u,
\end{aligned}
$$

for $n=0,1,2, \cdots$.

Proof. The result is readily obtained by the same argument used in the previous section, $S_{n}(u)$ being given by (5.6).

When $\alpha=\beta,(7.1)$ becomes

$$
\begin{aligned}
a_{n}= & \frac{(-1)^{n} \pi^{1 / 2}(2 n+2 \alpha+1) \Gamma(n+2 \alpha+1)}{(b-a)^{\alpha+1 / 2} \Gamma(n+\alpha+1)} \\
& \times \int_{0}^{\infty} f(u) u^{-\alpha-1 / 2} e^{-(b+a) u / 2} I_{n+\alpha+1 / 2}\left(\frac{b-a}{2} u\right) d u .
\end{aligned}
$$

The expression (7.2) was previously obtained by Wimp [2] although his method of derivation is somewhat different. Since $I_{m}(u)=e^{-m \times i / 2} J_{m}(i u)$, the integral in (7.2) is of Hankel type (see [11]). Moreover, when $b+a \neq 0$, the integral may also be reduced to known Laplace transforms. Thus, in many cases, with the aid of tables of integral transforms [10], [11], the coefficients $a_{n}$ may be immediately determined. Various functions satisfying the conditions of Theorem 4 have been considered in [2] and we refer the reader to this paper for examples.

8. The Stieltjes Transform Technique. In the Stieltjes transform technique, we shail derive an alternative expression for the coefficients of a function $F(t)$ belonging to the class $B[s ;(a, b)]$, i.e., $F(t)$ is such that

$$
F(t)=\delta[f(u)]=\int_{0}^{\infty} \frac{f(u)}{u+t} d t
$$

for $a<t<b$. The requirement that $F(t)$ should belong to $B[S ;(a, b)]$ implies that $F(t)$, considered as a function of a complex variable $t$, must be analytic in the complex $t$-plane cut along the negative real axis (see [22, p. 328]). In other words, $F(t)$ can only be represented by the integral in (8.1) for all $t$ for which $|\arg t|<\pi$. For this reason, it is only possible to consider the expansion of such $F(t)$ over an interval $(a, b)$, where $0 \leqq a<b$. We shall give here the fundamental result and a generalisation of the technique in a particular case.

We have, from [18, 110.8 , Eqs. (18) and (20)], 


$$
\begin{aligned}
S_{n}(u)= & \int_{a}^{b} \frac{(b-t)^{\alpha}(t-a)^{\beta} P_{n}^{(\alpha, \beta)}(t)}{t+u} d t \\
= & (-1)^{n}(b-a)^{n+\alpha+\beta+1} \frac{\Gamma(n+\alpha+1) \Gamma(n+\beta+1)}{\Gamma(2 n+\alpha+\beta+2)} \\
& \times(u+a)^{-n-1}{ }_{2} F_{1}\left(n+1, n+\beta+1 ; 2 n+\alpha+\beta+2 ;-\frac{b-a}{u+a}\right),
\end{aligned}
$$

where ${ }_{2} F_{1}(k, l ; m ; u)$ denotes a hypergeometric function.

Making use of (8.2) and employing an argument similar to that described in Section 6, we may prove

THEOREM 5. Let $F(t)$ be a function such that

(i) $F(t) \in B[s ;(a, b)]$, where $0 \leqq a<b$ and $F(t)=s[f(u)]$.

(ii) $f(u)(u+t)^{-1}(b-t)^{\alpha}(t-a)^{\beta} \odot_{n}^{(\alpha, \beta)}(t) \in C[(a, b) ;(0, \infty)]$.

Then the Jacobi coefficients for $F(t)$ are defined by

$$
\begin{aligned}
a_{n}=(-1)^{n}(b-a)^{n} & \frac{\Gamma(n+1) \Gamma(n+\alpha+\beta+1)}{\Gamma(2 n+\alpha+\beta+1)} \\
& \times \int_{0}^{\infty} f(u)(u+a)^{-n-1} \\
& \quad \cdot{ }_{2} F_{1}\left(n+1, n+\beta+1 ; 2 n+\alpha+\beta+2 ;-\frac{b-a}{u+a}\right) d u,
\end{aligned}
$$

for $n=0,1,2, \cdots$.

The expression (8.3) does not reduce to any simpler form with the exception of the case in which $\alpha=\beta=-\frac{1}{2}$. (Expansion in series of shifted Chebyshev polynomials of the first kind $J_{n}(t)=T_{n}(2 t /(b-a)-(b+a) /(b-a))$, for $a \leqq t \leqq b$.) In this case, the hypergeometric function ${ }_{2} F_{1}\left(n+1, n+\frac{1}{2} ; 2 n+1 ;-(b-a) /(u+a)\right)$ becomes an elementary function [19, p. 101, Eq. (5)]. The Chebyshev coefficients may be deduced from (8.3) to be

$$
a_{n}=2(-1)^{n}(b-a)^{n} \int_{0}^{\infty} \frac{f(u)}{(u+a)^{n+1}}\left(1+\frac{b-a}{u+a}\right)^{-1 / 2}\left[1+\left(1+\frac{b-a}{u+a}\right)^{1 / 2}\right]^{-2 n} d u
$$

which, on putting $(u+a) /(b-a)=\sinh ^{2} v$ and applying the inversion theorem for Stieltjes transform [22], becomes (with $a=0, b=1$ )

$$
a_{n}=\frac{2 i(-1)^{n}}{\pi} \int_{0}^{\infty}\left[F\left(e^{i \pi} \sinh ^{2} v\right)-F\left(e^{-i \pi} \sinh ^{2} v\right)\right] e^{-2 n v} d v
$$

The expression (8.4) was previously obtained by Miller [7]; however, his method of derivation was based on the theory of functions of a complex variable, and the relation of this result to the Stieltjes transform was not considered.

We shall next consider a generalisation of the Stieltjes transform technique in the particular case of Chebyshev coefficients. The above technique may be extended to give the Chebyshev coefficients for a function of the form $G(t)=p_{m}(t) F(t)$, where $p_{m}(t)$ is a polynomial of degree $m$, and $F(t)$ is a function of the class $B[s ;(a, b)]$. For simplicity, we shall assume that $a=0$ and $b=1$, and the Chebyshev coefficients which we wish to determine are defined by 


$$
a_{n}=\frac{1}{\pi} \int_{0}^{1} \frac{G(t) T_{n}^{*}(t)}{t^{1 / 2}(1-t)^{1 / 2}} d t,
$$

where $T_{n}^{*}(t)=T_{n}(2 t-1)$ for $0 \leqq t \leqq 1$.

We first prove a lemma.

LEMMA 2. If, for integers $n \geqq m \geqq 0$, we define

$$
\begin{aligned}
v_{n, m}(t) & =T_{n}^{*}(t) p_{m}(t) t^{-1 / 2}(1-t)^{-1 / 2} & & (0<t<1), \\
& =0 & & (t>1),
\end{aligned}
$$

where $p_{m}(t)$ is a polynomial of degree $m$, then the Stieltjes transform $V_{n, m}(u)$ of $v_{n, m}(t)$ is given by

$$
V_{n, m}(u)=\pi(-1)^{n} \frac{\left[(u+1)^{1 / 2}-u^{1 / 2}\right]}{u^{1 / 2}(u+1)^{1 / 2}} p_{m}(-u) .
$$

Proof. Consider first the integral

$$
S_{n, m}(u)=\int_{0}^{1} \frac{T_{n}^{*}(t) T_{m}^{*}(t)}{t^{1 / 2}(1-t)^{1 / 2}(t+u)} d t .
$$

For all integers $n \geqq m \geqq 0$, we have

$$
2 T_{n}^{*}(t) T_{m}^{*}(t)=T_{n+m}^{*}(t)+T_{n-m}^{*}(t),
$$

so that we can write $S_{n, m}(u)$ as

$$
S_{n, m}(u)=\frac{1}{2}\left[S_{n+m}(u)+S_{n-m}(u)\right],
$$

where

$$
S_{k}(u)=\int_{0}^{1} \frac{T_{k}^{*}(t) d t}{t^{1 / 2}(1-t)^{1 / 2}(t+u)},
$$

for $k \geqq 0 . S_{k}(u)$ may be deduced from (8.2) to be

$$
S_{k}(u)=(-1)^{k} \pi \frac{\left[(u+1)^{1 / 2}-u^{1 / 2}\right]^{2 k}}{u^{1 / 2}(u+1)^{1 / 2}} .
$$

We find from (8.7) and (8.9) that

$$
\begin{aligned}
S_{n, m}(u)= & \pi(-1)^{n} \frac{\left[(u+1)^{1 / 2}-u^{1 / 2}\right]^{2 n}}{u^{1 / 2}(u+1)^{1 / 2}} \\
& \times \frac{(-1)^{m}}{2}\left\{\left[(u+1)^{1 / 2}-u^{1 / 2}\right]^{2 m}+\left[(u+1)^{1 / 2}+u^{1 / 2}\right]^{2 m}\right\} .
\end{aligned}
$$

Making use of the definition of the shifted Chebyshev polynomials for an argument outside $[0,1]$, i.e.,

$$
T_{a}^{*}(u)=\frac{1}{2}\left\{\left[u^{1 / 2}+(u-1)^{1 / 2}\right]^{2 Q}+\left[u^{1 / 2}-(u-1)^{1 / 2}\right]^{2 a}\right\},
$$

for $q=0,1,2, \cdots$, we may write $(8.10)$ as

$$
S_{n, m}(u)=\pi(-1)^{n} u^{-1 / 2}(1+u)^{-1 / 2}\left[(u+1)^{1 / 2}-u^{1 / 2}\right]^{2 n} T_{m}^{*}(-u) .
$$

Since $p_{m}(t)$ may be expressed as 


$$
p_{m}(t)=\sum_{k=0}^{m} c_{k, m} T_{k}^{*}(t)
$$

the Stieltjes transform $V_{n, m}(u)$ is given by

$$
V_{n, m}(u)=\sum_{k=0}^{m} c_{k, m} S_{n, k}(u) .
$$

The result follows immediately on making use of (8.11).

We can now state and prove the theorem.

THEOREM 6. Let $G(t)$ be a function of the form

$$
G(t)=p_{m}(t) F(t),
$$

where $p_{m}(t)$ is a polynomial of degree $m$ and $F(t)$ satisfies the following conditions:

(i) $F(t) \in B[s ;(0,1)]$, where $F(t)=s[f(u)]$.

(ii) $p_{m}(t) f(u)(u+t)^{-1} t^{-1 / 2}(1-t)^{-1 / 2} T_{n}^{*}(t) \in C[(0,1) ;(0, \infty)]$.

Then the Chebysheo coefficients for $G(t)$ are defined by

$$
a_{n}=4(-1)^{n} \int_{0}^{\infty} f\left(\sinh ^{2} v\right) p_{m}\left(-\sinh ^{2} v\right) e^{-2 n v} d v,
$$

for $n \geqq m \geqq 0$.

Proof. From conditions (i), (ii), and (8.5) the Chebyshev coefficients for $G(t)$ may be written as

$$
a_{n}=\frac{2}{\pi} \int_{0}^{\infty} f(u) d u \int_{0}^{1} \frac{v_{n, m}(t)}{t+u} d t
$$

which, by Lemma 2, becomes

$$
a_{n}=2(-1)^{n} \int_{0}^{\infty} \frac{\left[(u+1)^{1 / 2}-u^{1 / 2}\right]^{2 n}}{u^{1 / 2}(u+1)^{1 / 2}} f(u) p_{m}(-u) d u, \quad(n \geqq m \geqq 0) .
$$

Putting $u=\sinh ^{2} v$ in (8.14) we obtain the required Eq. (8.13).

As an illustration of Theorem 6 , let us consider the function

$$
G(t)=t_{2}^{p} F_{1}\left(1, w ; 1+\lambda ;-\frac{1}{t}\right)
$$

where $p$ is an integer greater than or equal to $-1, p+w>-\frac{1}{2}$, and $\lambda-w>1$. It can be readily verified that the function

$$
F(t)=\frac{1}{t}{ }_{2} F_{1}\left(1, w ; 1+\lambda ;-\frac{1}{t}\right)
$$

is the Stieltjes transform of

$$
\begin{aligned}
f(u) & =\frac{\Gamma(\lambda+1)}{\Gamma(\lambda-w+1) \Gamma(w)}(1-u)^{\lambda-w} u^{w-1} & & (0<u<1), \\
& =0 & & (u>1),
\end{aligned}
$$

(see [19, p. 59, Eq. (10)]). Thus, $G(t)$ is of the required form (8.12) with $p_{m}(t)=t^{p+1}$. The verification of condition (ii) is rather cumbersome since the function 


$$
\phi(t, u)=(u+t)^{-1} p_{m}(t) f(u) t^{-1 / 2}(1-t)^{-1 / 2} T_{n}^{*}(t)
$$

is infinite at the endpoints of both ranges. However, repeated applications of the theorems in Titchmarsh [20, Sections 1.84 and 1.85] show that $\phi(t, u)$ is indeed in $C[(0,1) ;(0,1)]$. Thus, we can apply Theorem 6 to the function $G(t)$ defined by $(8.15)$ and obtain

$$
a_{n}=\frac{4(-1)^{n+p+1} \Gamma(\lambda+1)}{\Gamma(w) \Gamma(\lambda-w+1)} \int_{0}^{v_{0}} e^{-2 n v}(\sinh v)^{2(p+w)}\left(1-\sinh ^{2} v\right)^{\lambda-w} d v,
$$

where $\sinh v_{0}=1$. The evaluation of this integral depends on the values of $p, \lambda$, and $w$.

Let us first note a special case; with $p=-1, \lambda=w=1$, the function $G(t)=$ $\log (1+1 / t)$, and we have

$$
a_{n}=\frac{2(-1)^{n+1}}{n}\left[(\sqrt{ } 2+1)^{-2 n}-1\right]
$$

for $n=1,2,3, \cdots$ with $a_{0}=4 \log (\sqrt{ } 2+1)$.

If we now assume that $p+w>0$ and $\lambda-w>0$, then we can use Laplace's method (see, for example, [23]) for approximating the integral in (8.16) for large $n$. We find after some algebra

$$
a_{n} \sim \frac{4(-1)^{n+p+1} \pi^{1 / 2} \Gamma(\lambda+1)(p+w)^{2(p+w)+1 / 2} e^{-2(p+w)}}{\Gamma(w) \Gamma(\lambda-w+1) n^{2(p+w)+1}}
$$

for large $n$.

Acknowledgements. This work was done while one of the authors (P.D. Tuan) was in receipt of a University of Tasmania Research Scholarship. The authors are indebted to the referee for his valuable comments on a previous draft of this paper.

Mathematics Department

University of Tasmania

Hobart, Tasmania, Australia

1. G. SANSONE, Orthogonal Functions, Zanichelli, Bologna, 1952; English transl., Pure and Appl. Math., vol. 9, Interscience, New York, 1959. MR 13, 741; MR 21 \#2140.

2. J. WIMP, "Polynomial approximations to integral transforms," Math. Comp., v. 15, 1961, pp. 174-178.

3. C. W. Clenshaw, Chebyshev Series for Mathematical Functions, National Physical Lab. Math. Tables, vol. 5, Department of Scientific and Industrial Research, HMSO, London, 1962. MR 26 \#362.

4. Y. L. LUKE \& J. WIMP, "Jacobi polynomial expansions of a generalised hypergeometric function over a semi-infinite ray," Math. Comp., v. 17, 1963, pp. 395-404. MR 28 \#255.

5. D. Elliotr, "The evaluation and estimation of the coefficients in the Chebyshev series expansion of a function," Math. Comp., v. 18, 1964, pp. 274-284. MR 29 \#176.

6. D. Elliotr \& G. Szekeres, "Some estimates of the coefficients in the Chebyshev series expansion of a function," Math. Comp., v. 19, 1965, pp. 25-32. MR 30 \#2666.

7. G. F. MILlER, "On the convergence of the Chebyshev series for functions possessing a singularity in the range of representation," SIAM J. Numer. Anal., v. 3, 1966, pp. 390-409. MR 34 \#3165.

8. J. WiMP, "The asymptotic representation of a class of $G$-functions for large parameter," Math. Comp., v. 21, 1967, pp. 639-646. MR 36 \#6665.

9. Y. L. LUKE, The Special Functions and their Approximations. Vol. 1, Academic Press, New York and London, 1969. MR 39 \#3039. 
10. A. ERdélyi et al., Tables of Integral Transforms. Vol. 1, McGraw-Hill, New York, 1954. MR 15, 868.

11. A. ERdÉlyi et al., Tables of Integral Transforms. Vol. 2, McGraw-Hill, New York, 1954. MR 16, 468.

12. E. C. Titchmarsh, Introduction to the Theory of Fourier Integrals, Clarendon Press, Oxford, 1937

13. P. D. Tuan, On the Estimation of Fourier Coefficients, Ph.D. Thesis, University of Tasmania, Tasmania, 1969.

14. L. J. Slater, Confluent Hypergeometric Functions, Cambridge Univ. Press, New York, 1960. MR 21 \# 5753.

15. D. S. Jones, Generalised Functions, McGraw-Hill, New York, 1966. MR 36 \#623.

16. M. Abramowitz \& I. A. STEgUN (Editors), Handbook of Mathematical Functions

With Formulas, Graphs, and Mathematical Tables, Nat. Bur. Standards Appl. Math. Series, 55, Superintendent of Documents, U.S. Government Printing Office, Washington, D. C., 1964. MR 29 \#4914.

17. W. R. LE PAge, Complex Variables and the Laplace Transform for Engineers, Internat. Series in Pure and Appl. Math., McGraw-Hill, New York, 1961. MR 22 \#8293.

18. A. ERdély ET AL., Higher Transcendental Functions. Vol. 2, McGraw-Hill, New York, 1953. MR 15, 419.

19. A. ERDÉLYI ET AL., Higher Transcendental Functions. Vol. 1, McGraw-Hill, New York, 1953. MR 15, 419.

20. E. C. Trtchmarsh, The Theory of Functions, Clarendon Press, Oxford, 1932.

21. S. SAKs, Theory of the Integral, Monografie Mat., vol. 7, PWN, Warsaw, 1937.

22. D. V. WIDDER, The Laplace Transform, Princeton Math. Series, vol. 6, Princeton Univ. Press, Princeton, N. J., 1941. MR 3, 232.

23. N. G. DE BRUIJN, Asymptotic Methods in Analysis, Bibliotheca Math., vol. 4, NorthHolland, Amsterdam; Noordhoff, Groningen; Interscience, New York, 1958. MR 20 \#6003. 